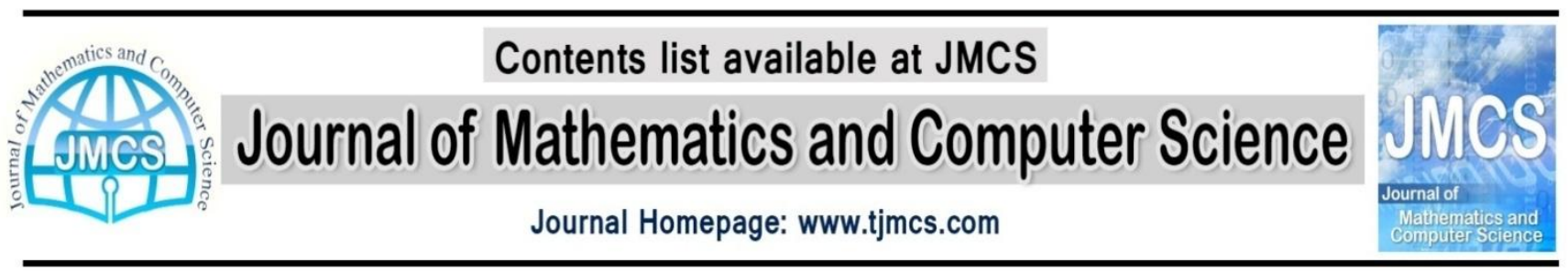

\title{
FIXED POINTS OF NONEXPANSIVE MAPPINGS IN BANACH SPACES
}

\author{
F. Golkar, A. Dianatifar, A. M. Aminpour, M. Sadeghi \\ Department of Mathematics, Shahid Chamran university of Ahvaz, Ahvaz, Iran \\ E-mail \\ f-golkar@phdstu.scu.ac.ir \\ Dianati.math@gmail.com \\ aminpour@scu.ac.ir \\ m10sadeghi@gmail.com
}

Article history:

Received July 2014

Accepted August 2014

Available online August 2014

\section{Abstract}

In this paper we study the approximation of common fixed points of a finite family of nonexpansive mappings in uniformly smooth Banach spaces. Also we show that the convergence of the proposed algorithm can be proved under some types of control conditions.

Keywords: Nonexpansive mapping, Strong convergence, Common fixed point, Uniformly smooth Banach space.

\section{Introduction}

Let $\mathrm{X}$ be a real Banach space. A mapping $T: X \rightarrow X$ is said to be nonexpansive provided that

$$
\|T x-T y\| \leq\|x-y\|, \quad x, y \in X .
$$

Let $T_{i}, i=1,2, \ldots, N$ be a finite family of nonexpansive self-mappings on $X$, and let $F i x\left(T_{i}\right)=\left\{x \in X: T_{i} x=x\right\}$. We shall assume that $F:=\bigcap_{i=1}^{N} F i x\left(T_{i}\right) \neq \varnothing$. Assume that for each natural number $\mathrm{n}$, there correspond $\mathrm{N}$ real numbers $\alpha_{n, 1}, \alpha_{n, 2}, \ldots, \alpha_{n, N}$ in the half-open interval $(0,1]$. Given $\mathrm{N}$ self-mappings $T_{1}, T_{2}, \ldots, T_{N}$ as in [4] one can define, for each $\mathrm{n}$ the mappings $U_{n, 1}, U_{n, 2}, \ldots, U_{n, N}$ in the following way: 


$$
\begin{aligned}
U_{n, 1} & =\alpha_{n, 1} T_{1}+\left(1-\alpha_{n, 1}\right) I \\
\mathrm{U}_{n, 2} & =\alpha_{n, 2} \mathrm{~T}_{2} \mathrm{U}_{n, 1}+\left(1-\alpha_{n, 2}\right) \mathrm{I} \\
& \cdot \\
& \cdot \\
U_{n, N-1} & =\alpha_{n, N-1} T_{n-1} U_{n, N-2}+\left(1-\alpha_{n, N-1}\right) I \\
W_{n}: & =U_{n, N}=\alpha_{n, N} T_{N} U_{n, N-1}+\left(1-\alpha_{n, N}\right) I
\end{aligned}
$$

Nonexpansivity of $T_{i}$ yields the nonexpansivity of $W_{n}$. Moreover, in [4], it is shown that Fix $\left(W_{n}\right)=F$.

In the case $X$ equals a real Hilbert space H, Y. Yao in [8] introduced an iterative algorithm to approximate the common fixed points of a finite family of nonexpansive self-mappings defined on the real Hilbert space H. To write down Yao's proposed scheme, we recall that $f: H \rightarrow H$ is said to be a contraction if there exists a number $0<\alpha<1$ such that

$$
\|f(x)-f(y)\| \leq \alpha\|x-y\|, \quad x, y \in H .
$$

Recall also that a bounded linear operator $A$ on the Hilbert space $\mathrm{H}$ is said to be strongly positive if there exists a positive number $\bar{\gamma}$ such that

$$
\langle A x, x\rangle \geq \vec{\gamma}\|x\|^{2}, \quad x \in H .
$$

Assuming that $I$ denotes the identity operator on $\mathrm{H}$ and $\left\{\lambda_{n}\right\}$ is a sequence of real numbers, and $u_{0} \in H$ is arbitrarily chosen, Yao introduced

$$
u_{n+1}=\alpha_{n} \gamma f\left(u_{n}\right)+\beta u_{n}+\left((1-\beta) I-\lambda_{n} A\right) W_{n} u_{n}
$$

Where $\gamma, \beta$ are two positive real numbers such that $\beta<1, f: H \rightarrow H$ is a contraction with coefficient $0<\alpha<1, A$ is a strongly positive bounded linear operator with coefficient $\bar{\gamma}>0$ such that $0<\gamma<\frac{\bar{\gamma}}{\alpha}$ and $W_{n}$ is the self-mapping of $\mathrm{H}$ generated by (1.1).

Under the assumption that the sequence $\left\{\lambda_{n}\right\}$ satisfies the following two control conditions $\left(C_{1}\right): \lim _{n \rightarrow \infty} \lambda_{n}=0$,

$\left(C_{2}\right): \sum_{n=1}^{\infty} \lambda_{n}=\infty$,

Yao proved that the iterative sequence $\left\{u_{n}\right\}$ converges strongly to the unique solution of the variational inequality

$$
\left\langle(A-\gamma f) u^{*}, u-u^{*}\right\rangle \geq 0 \quad u \in F
$$

Let $X$ be a real Banach space. For $\mathrm{t}>0$, we define

$$
\rho(\mathrm{t})=\frac{1}{2} \sup \{\|\mathrm{x}+\mathrm{ty}\|+\|\mathrm{x}-\mathrm{ty}\|-2:\|x\|=\|y\|=1\} .
$$

A Banach space $X$ is said to be uniformly smooth if

$$
\lim _{t \rightarrow 0} \frac{\rho(t)}{t}=0 .
$$


Geometrically, this means that the function $x \mapsto h(x)=\|x\|$ is uniformly Ferechetdifferentiable on the unit sphere, that is,

$$
\lim _{t \rightarrow 0^{+}\|x\|=\|y\|=1} \sup _{\| x=1}\left|\frac{\|x+t y\|-\|x\|}{t}-\left\langle h^{\prime}(x), y\right\rangle\right|=0 .
$$

Let $X$ be a real Banach space. Recall the (normalized) duality mapping $J$ from $X$ to $X^{*}$ the dual space of $X$, is given by

$$
J(x)=\left\{x^{*} \in X^{*}:\left\langle x, x^{*}\right\rangle=\|x\|^{2}=\left\|x^{*}\right\|^{2}\right\}
$$

In this paper we assume that $X$ is a uniformly smooth Banach space and $C$ is a closed convex subset of $X$. We further assume that $f: C \rightarrow C$ is a contraction with coefficient $0<\alpha<1$. By a slight modification of the sequence introduced by Yao, and replacing the strongly positive operator $A$ by the identity operator $I$ acting instead on a uniformly smooth Banach space $X$, we shall see that the sequence

$$
u_{n+1}=\lambda_{n} f\left(u_{n}\right)+\beta u_{n}+\left(1-\beta-\lambda_{n}\right) W_{n} u_{n}
$$

Converges strongly to the unique solution of the variational inequality

$$
\left\langle(I-f) u^{*}, u-u^{*}\right\rangle \geq 0 \quad u \in F .
$$

Here $0<\beta<1$, and $\mathrm{W}_{n}$ 's are the self-mappings of $C$ generated by (1.1), moreover the sequence $\left\{\lambda_{n}\right\}$ satisfies the control conditions $\left(C_{1}\right),\left(C_{2}\right)$.

\section{PRELIMINARIES}

This section collects some lemmas which will be used in the proofs for the main results in the next section.

Lemma 2.1. [7] Let $\left\{x_{n}\right\}$ and $\left\{y_{n}\right\}$ be bounded sequences in a Banach space $X$ and let $\left\{\alpha_{n}\right\}$ be a sequence in $[0,1]$ with $0<\liminf _{n \rightarrow \infty} \alpha_{n} \leq \limsup _{n \rightarrow \infty} \alpha_{n}<1$. Suppose $x_{n+1}=\alpha_{n} x_{n}+\left(1-\alpha_{n}\right) y_{n}$ for all integers $n \geq 0$ and

$\underset{n \rightarrow \infty}{\limsup }\left(\left\|y_{n+1}-y_{n}\right\| \dashv x_{n+1}-x_{n} \| \quad\right) \leq 0$. Then, $\lim _{n \rightarrow \infty}\left\|y_{n}-x_{n}\right\|=0$.

Lemma 2.2. [6] Let $X$ be a real Banach space. Then for all $x, y \in X$

$$
\|x+y\|^{2} \leq\|x\|^{2}+2\langle y, J(x+y)\rangle
$$

Lemma 2.3. [1] Assume $\left\{a_{n}\right\}$ to be a sequence of nonnegative real numbers such that

$$
a_{n+1} \leq\left(1-\gamma_{n}\right) a_{n}+\delta_{n} \gamma_{n}, \quad \forall n \geq 0
$$

where $\left\{\gamma_{n}\right\}$ is a sequence in $(0,1)$ and $\left\{\delta_{n}\right\}$ is a sequence in $R$ such that

(i) $\sum_{n=0}^{\infty} \gamma_{n}=\infty$

(ii) either $\limsup _{n \rightarrow \infty} \delta_{n} \leq 0$ or $\sum_{n=0}^{\infty}\left|\delta_{n} \gamma_{n}\right|<\infty$. 
Then $\lim _{n \rightarrow \infty} a_{n}=0$.

Proposition2.4. [3] If $X$ is a uniformly smooth Banach space, then the normalized duality mapping $J$ defined by (1.2) is single-valued and uniformly continuous on each bounded subset of $X$ from the norm topology of $X$ to the norm topology of $X^{*}$.

Lemma 2.5. [2] Let $X$ be a uniformly smooth Banach space, $C$ a closed convex subset of $X$, $T: C \rightarrow C$ a nonexpansive mapping with $\operatorname{Fix}(T) \neq \varnothing$, and $f: C \rightarrow C$ be a contraction. then $\left\{x_{t}\right\}$ defined by

$$
x_{t}=t f\left(x_{t}\right)+(1-t) T x_{t}
$$

Convergence strongly to a point in $\operatorname{Fix}(T)$.

\section{Main results}

We now state and prove the main result of this paper.

Theorem 3.1. Let $C$ be a nonempty closed convex subset of a uniformly smooth Banach space $X, f$ be a contraction on $C$ with coefficient $0<\alpha<1$, choose any $u_{0} \in C$. Define $\left\{u_{n}\right\}$ by

$$
u_{n+1}=\lambda_{n} f\left(u_{n}\right)+\beta u_{n}+\left(1-\beta-\lambda_{n}\right) W_{n} u_{n}
$$

Where $\beta$ is a positive real number such that $\beta<1$ and $W_{n}$ is the self-mapping of $C$ generated by (1.1), suppose the sequence $\left\{\alpha_{n, i}\right\}$ satisfy $\lim _{n \rightarrow \infty}\left(\alpha_{n, i}-\alpha_{n-1, i}\right)=0$ and the sequence $\left\{\lambda_{n}\right\}$ satisfying the control conditions $\left(C_{1}\right),\left(C_{2}\right)$. Then the sequence $\left\{u_{n}\right\}$ converges strongly to the unique solution of the variational inequality defined by (1.3).

Proof. First we observe that $\left\{u_{n}\right\}$ is bounded. Indeed, pick any $p \in F$ to obtain

$$
\begin{aligned}
\left\|u_{n+1}-p\right\| & \leq \lambda_{n}\left\|f\left(u_{n}\right)-f(p)\right\|+\beta\left\|u_{n}-p\right\|+\left(1-\beta-\lambda_{n}\right)\left\|W_{n} u_{n}-p\right\|+\lambda_{n}\|f(p)-p\| \\
& \leq\left(\lambda_{n} \alpha+1-\lambda_{n}\right)\left\|u_{n}-p\right\|+\lambda_{n}\|f(p)-p\| \\
& =\left[1-\lambda_{n}(1-\alpha)\right]\left\|u_{n}-p\right\|+\lambda_{n}\|f(p)-p\|
\end{aligned}
$$

It follows from (1.3) by induction that

$$
\left\|u_{n}-p\right\| \leq \max \left\{\left\|u_{0}-p\right\|, \frac{\|f(p)-p\|}{1-\alpha}\right\}
$$

Hence, $\left\{u_{n}\right\}$ is bounded, and so are $\left\{f\left(u_{n}\right)\right\}$ and $\left\{W_{n} u_{n}\right\}$.

Next, we claim that $\lim _{n \rightarrow \infty}\left\|u_{n+1}-u_{n}\right\|=0$.

Define

$$
u_{n+1}=(1-\beta) z_{n}+\beta u_{n}
$$

We shall use $\mathrm{M}$ to denote the possible different constants appearing in the following reasoning. Observe that from the definition of $z_{n}$, we obtain 


$$
\begin{aligned}
z_{n+1}-z_{n} & =\frac{u_{n+2}-\beta u_{n+1}}{1-\beta}-\frac{u_{n+1}-\beta u_{n}}{1-\beta} \\
& =\frac{\lambda_{n+1} f\left(u_{n+1}\right)+\left(1-\beta-\lambda_{n+1}\right) W_{n+1} u_{n+1}}{1-\beta}-\frac{\lambda_{n} f\left(u_{n}\right)+\left(1-\beta-\lambda_{n}\right) W_{n} u_{n}}{1-\beta} \\
& =\frac{\lambda_{n+1} f\left(u_{n+1}\right)-\frac{\lambda_{n}}{1-\beta} f\left(u_{n}\right)+W_{n+1} u_{n+1}-W_{n} u_{n}+\frac{\lambda_{n}}{1-\beta} W_{n} u_{n}-\frac{\lambda_{n+1}}{1-\beta} W_{n+1} u_{n+1}}{1-\beta} \\
& =\frac{\lambda_{n+1}}{1-\beta}\left[f\left(u_{n+1}\right)-W_{n+1} u_{n+1}\right]+W_{n+1} u_{n+1}+\frac{\lambda_{n}}{1-\beta}\left[W_{n} u_{n}-f\left(u_{n}\right)\right]-W_{n} u_{n} \pm W_{n+1} u_{n} .
\end{aligned}
$$

It follows that

$$
\begin{aligned}
\left\|z_{n+1}-z_{n}\right\|-\left\|u_{n+1}-u_{n}\right\| \leq & \frac{\lambda_{n+1}}{1-\beta}\left(\| f\left(u_{n+1}\|+\| W_{n+1} u_{n+1} \|\right)\right. \\
& +\frac{\lambda_{n}}{1-\beta}\left(\left\|f\left(u_{n}\right)\right\|+\left\|W_{n} u_{n}\right\|\right)+\left\|W_{n+1} u_{n+1}-W_{n+1} u_{n}\right\| \\
& +\left\|W_{n+1} u_{n}-W_{n} u_{n}\right\|-\left\|u_{n+1}-u_{n}\right\| \\
& \leq \frac{\lambda_{n+1}}{1-\beta}\left(\| f\left(u_{n+1}\|+\| W_{n+1} u_{n+1} \|\right)\right. \\
& +\frac{\lambda_{n}}{1-\beta}\left(\left\|f\left(u_{n}\right)\right\|+\left\|W_{n} u_{n}\right\|\right)+\left\|W_{n+1} u_{n}-W_{n} u_{n}\right\|
\end{aligned}
$$

From (1.1), since $T_{N}$ and $U_{n, N}$ are nonexpansive,

$$
\begin{aligned}
\left\|W_{n+1} u_{n}-W_{n} u_{n}\right\|-\| u_{n+1}-u_{n} & \| \\
& =\left\|\alpha_{n+1, N} T_{N} U_{n+1, N-1} u_{n}+\left(1-\alpha_{n+1, N}\right) u_{n}-\alpha_{n, N} T_{N} U_{n, N-1} u_{n}-\left(1-\alpha_{n, N}\right) u_{n}\right\| \\
& \leq\left|\alpha_{n+1, N}-\alpha_{n, N}\right|\left\|u_{n}\right\|+\left\|\alpha_{n+1, N}\left(T_{N} U_{n+1, N-1} u_{n}-T_{N} U_{n, N-1} u_{n}\right)\right\| \\
& \leq 2 M\left|\alpha_{n+1, N}-\alpha_{n, N}\right|+\alpha_{n+1, N-1}\left\|U_{n+1, N-1} u_{n}-U_{n, N-1} u_{n}\right\|(3.3)
\end{aligned}
$$

Again, from (1.1),

$$
\begin{aligned}
\left\|U_{n+1, N-1} u_{n}-U_{n, N-1} u_{n}\right\| & =\mid \alpha_{n+1, N-1} T_{N-1} U_{n+1, N-2} u_{n}+\left(1-\alpha_{n+1, N-1}\right) u_{n} \| \\
& -\alpha_{n, N-1} T_{N-1} U_{n, N-2} u_{n}-\left(1-\alpha_{n, N-1}\right) u_{n} \\
& \leq\left|\alpha_{n+1, N-1}-\alpha_{n, N-1}\right|\left\|u_{n}\right\|+\left\|\alpha_{\mathrm{n}+1, \mathrm{~N}-1} U_{\mathrm{n}+1, \mathrm{~N}-2} u_{n}-\alpha_{n, N-1} T_{N-1} U_{n, N-2} u_{n}\right\| \\
& \leq\left|\alpha_{n+1, N-1}-\alpha_{n, N-1}\right|\left\|u_{n}\right\|+\alpha_{n+1, N-1}\left\|U_{n+1, N-2} u_{n}-U_{n, N-2} u_{n}\right\| \\
& +\left|\alpha_{\mathrm{n}+1, \mathrm{~N}-1}-\alpha_{\mathrm{n}, \mathrm{N}-1}\right|\left\|T_{\mathrm{N}-1} U_{\mathrm{n}, \mathrm{N}-2} u_{n}\right\| \\
& \leq 2 M\left|\alpha_{n+1, N-1}-\alpha_{n, N-1}\right|+\left\|U_{n+1, N-2} u_{n}-U_{n, N-2} u_{n}\right\|
\end{aligned}
$$

Therefore, we have

$$
\begin{aligned}
\left\|U_{n+1, N-2} u_{n}-U_{n, N-2} u_{n}\right\| & \leq 2 M\left|\alpha_{n+1, N-1}-\alpha_{n, N-1}\right|+2 M\left|\alpha_{n+1, N-2}-\alpha_{n, N-2}\right| \\
& \leq 2 M \sum_{i=2}^{N-1}\left|\alpha_{n+1, i}-\alpha_{n, i}\right|+\left\|U_{n+1,1} u_{n}-U_{n, 1} u_{n}\right\| \\
& =\left\|\alpha_{n+1,1} T_{1} u_{n}+\left(1-\alpha_{\mathrm{n}+1,1}\right)-\alpha_{n, 1} T_{1} u_{n}-\left(1-\alpha_{n, 1}\right) u_{n}\right\|+2 M \sum_{i=2}^{N-1}\left|\alpha_{n+1, i}-\alpha_{n, i}\right|
\end{aligned}
$$




$$
\begin{aligned}
& \leq\left|\alpha_{n+1,1}-\alpha_{n, 1}\right|\left\|u_{n}\right\|+\left\|\alpha_{n+1,1} T_{1} u_{n}-\alpha_{n, 1} T_{1} u_{n}\right\|+2 M \sum_{i=2}^{N-1}\left|\alpha_{n+1, i}-\alpha_{n, i}\right| \\
& \leq 2 M \sum_{i=1}^{N-1}\left|\alpha_{n+1, i}-\alpha_{n, i}\right|
\end{aligned}
$$

Substituting (3.5) into (3.3), we have

$$
\begin{aligned}
\left\|W_{n+1} u_{n}-W_{n} u_{n}\right\| & \leq 2 M\left|\alpha_{n+1, N}-\alpha_{n, N}\right|+2 \alpha_{n+1, N} M \sum_{i=1}^{N-1}\left|\alpha_{n+1, i}-\alpha_{n, i}\right| \\
& \leq 2 M\left|\alpha_{n+1, N}-\alpha_{n, N}\right|+2 M \sum_{i=1}^{N-1}\left|\alpha_{n+1, i}-\alpha_{n, i}\right| \\
& =2 M \sum_{i=1}^{N}\left|\alpha_{n+1, i}-\alpha_{n, i}\right|
\end{aligned}
$$

Using (3.6) in (3.2), we get

$$
\begin{aligned}
\left\|z_{n+1}-z_{n}\right\|-\left\|u_{n+1}-u_{n}\right\| \leq & \frac{\lambda_{n+1}}{1-\beta}\left(\| f\left(u_{n+1}\|+\| W_{n+1} u_{n+1} \|\right)\right. \\
& +\frac{\lambda_{n}}{1-\beta}\left(\left\|f\left(u_{n}\right)\right\|+\left\|W_{n} u_{n}\right\|\right)+2 M \sum_{i=1}^{N}\left|\alpha_{n+1, i}-\alpha_{n, i}\right|
\end{aligned}
$$

Which implies that

$$
\limsup _{n \rightarrow \infty}\left(\left\|z_{n+1}-z_{n}\right\|-\left\|u_{n+1}-u_{n}\right\|\right) \leq 0
$$

We can obtain $\lim _{n \rightarrow \infty}\left\|z_{n}-u_{n}\right\|=0$ easily by lemma (2.1). Consequently

$$
\lim _{n \rightarrow \infty}\left\|u_{n+1}-u_{n}\right\|=\lim _{n \rightarrow \infty}(1-\beta)\left\|z_{n}-u_{n}\right\|=0
$$

We can write the sequence $\left\{u_{n}\right\}$ as follow

$$
\mathrm{u}_{\mathrm{n}+1}=\lambda_{n} \mathrm{f}\left(\mathrm{u}_{n}\right)-\lambda_{n} \mathrm{~W}_{n} \mathrm{u}_{n}+\mathrm{y}_{n}
$$

Where $y_{n}=W_{n} u_{n}+\beta\left(u_{n}-W_{n} u_{n}\right)$

Observing that $\mathrm{u}_{\mathrm{n}+1}-\mathrm{y}_{n}=\lambda_{n}\left(\mathrm{f}\left(\mathrm{u}_{n}\right)-\mathrm{W}_{n} \mathrm{u}_{n}\right)$, we can easily get

$$
\begin{gathered}
\lim _{n \rightarrow \infty}\left\|y_{n}-u_{n+1}\right\|=0 \\
\left\|u_{n}-y_{n}\right\| \leq\left\|u_{n}-u_{n+1}\right\|+\left\|u_{n+1}-y_{n}\right\|
\end{gathered}
$$

That is, $\lim _{n \rightarrow \infty}\left\|y_{n}-u_{n}\right\|=0$. On the other hand, we have

$$
\left\|W_{n} u_{n}-u_{n}\right\| \leq\left\|u_{n}-y_{n}\right\|+\left\|y_{n}-W_{n} u_{n}\right\| \leq\left\|u_{n}-y_{n}\right\|+\beta\left\|u_{n}-W_{n} u_{n}\right\|
$$

Which implies $(1-\beta)\left\|W_{n} u_{n}-u_{n}\right\| \leq\left\|u_{n}-y_{n}\right\|$ but we have $\beta \in(0,1)$ so we obtain

$$
\lim _{n \rightarrow \infty}\left\|W_{n} u_{n}-u_{n}\right\|=0
$$

Next, we claim that

$$
\limsup _{n \rightarrow \infty}\left\langle f(p)-p, J\left(u_{n+1}-p\right)\right\rangle \leq 0
$$

Let $u_{t}$ be the unique fixed point of the contraction mapping given by

$$
u \mapsto t f(u)+(1-t) W_{n} u
$$

Then, $u_{t}$ solves the fixed point equation $u_{t}=t f\left(u_{t}\right)+(1-t) W_{n} u_{t} \quad t \in(0,1)$. Thus 


$$
u_{t}-u_{n}=t\left(f\left(u_{t}\right)-u_{n}\right)+(1-t)\left(W_{n} u_{t}-u_{n}\right) .
$$

We apply Lemma 2.2 to get

$\left\|u_{t}-u_{n}\right\|^{2} \leq(1-t)^{2}\left\|W_{n} u_{t}-u_{n}\right\|^{2}+2 t\left\langle f\left(u_{t}\right)-u_{n}, J\left(u_{t}-u_{n}\right)\right\rangle$

$\leq\left(\left\|W_{n} u_{t}-W_{n} u_{n}\right\|+\left\|W_{n} u_{n}-u_{n}\right\|\right)^{2}+2 t\left\langle f\left(u_{t}\right)-u_{t}, J\left(u_{t}-u_{n}\right)\right\rangle+2 t\left\langle u_{t}-u_{n}, J\left(u_{t}-u_{n}\right)\right\rangle$

$\leq(1-t)^{2}\left(\left\|u_{t}-u_{n}\right\|^{2}+a_{n}(t)\right)+2 t\left\|u_{t}-u_{n}\right\|^{2}+2 t\left\langle f\left(u_{t}\right)-u_{t}, J\left(u_{t}-u_{n}\right)\right\rangle$

Where

$$
\mathrm{a}_{n}(\mathrm{t})=\left(2\left\|u_{t}-u_{n}\right\|+\left\|W_{n} u_{n}-u_{n}\right\|\right)\left\|W_{n} u_{n}-u_{n}\right\|
$$

Noting (3.7) $\lim _{n \rightarrow \infty} a_{n}(t)=0$

The last inequality (3.9) implies

It follows that

$$
\left\langle u_{t}-f\left(u_{t}\right), J\left(u_{t}-u_{n}\right)\right\rangle \leq \frac{t}{2}\left\|u_{t}-u_{n}\right\|^{2}+\frac{(1-t)^{2}}{2 t} a_{n}(t)
$$

$$
\limsup _{n \rightarrow \infty}\left\langle u_{t}-f\left(u_{t}\right), J\left(u_{t}-u_{n}\right)\right\rangle \leq \frac{t}{2} M^{2}
$$

Where $M>0$ is a constant such that $M \geq\left\|u_{t}-u_{n}\right\|$ for all $t \in(0,1)$ and $n \geq 1$. Letting $t \rightarrow 0$ in (3.11) and noting (3.10) yields

$$
\limsup _{n \rightarrow \infty}\left\langle u_{t}-f\left(u_{t}\right), J\left(u_{t}-u_{n}\right)\right\rangle \leq 0 .
$$

Moreover, we have that

$$
\begin{aligned}
\left\langle p-f(p), J\left(p-u_{n}\right)\right\rangle & =\left\langle p-f(p), J\left(p-u_{n}\right)-J\left(u_{t}-u_{n}\right)\right\rangle \\
& +\left\langle p-f(p)-u_{t}+f\left(u_{t}\right), J\left(u_{t}-u_{n}\right)\right\rangle+\left\langle u_{t}-f\left(u_{t}\right), J\left(u_{t}-u_{n}\right)\right\rangle \\
& =\left\langle p-f(p), J\left(p-u_{n}\right)-J\left(u_{t}-u_{n}\right)\right\rangle \\
& +\left\langle p-u_{t}, J\left(u_{t}-u_{n}\right)\right\rangle+\left\langle f\left(u_{t}\right)-f(p), J\left(u_{t}-u_{n}\right)\right\rangle \\
& +\left\langle u_{t}-f\left(u_{t}\right), J\left(u_{t}-u_{n}\right)\right\rangle
\end{aligned}
$$

Then, we obtain

$$
\begin{aligned}
\limsup _{n \rightarrow \infty}\left\langle p-f(p), J\left(p-u_{n}\right)\right\rangle & \leq \limsup _{n \rightarrow \infty}\left\langle p-f(p), J\left(p-u_{n}\right)-J\left(u_{t}-u_{n}\right)\right\rangle \\
& +\left\|p-u_{t}\right\| \limsup _{n \rightarrow \infty}\left\|u_{t}-u_{n}\right\|+\left\|f\left(u_{t}\right)-f(p)\right\| \limsup _{n \rightarrow \infty}\left\|u_{t}-u_{n}\right\| \\
& +\limsup _{n \rightarrow \infty}\left\langle u_{t}-f\left(u_{t}\right), J\left(u_{t}-u_{n}\right)\right\rangle \\
& \leq \limsup _{n \rightarrow \infty}\left\langle p-f(p), J\left(p-u_{n}\right)-J\left(u_{t}-u_{n}\right)\right\rangle \\
& +(1+\alpha)\left\|p-u_{t}\right\| \limsup _{n \rightarrow \infty}\left\|u_{t}-u_{n}\right\| \\
& +\limsup _{n \rightarrow \infty}\left\langle u_{t}-f\left(u_{t}\right), J\left(u_{t}-u_{n}\right)\right\rangle
\end{aligned}
$$

By Lemma 2.5, $u_{t} \rightarrow p \in F$ as $t \rightarrow 0$, which is the unique solution of the variational inequality (1.3). Noting proposition 2.4 , we obtain

$$
\limsup _{t \rightarrow 0} \limsup _{n \rightarrow \infty}\left\langle p-f(p), J\left(p-u_{n}\right)-J\left(u_{t}-u_{n}\right)\right\rangle=0
$$

Therefore we have 


$$
\begin{aligned}
& \limsup _{n \rightarrow \infty}\left\langle p-f(p), J\left(p-u_{n}\right)\right\rangle=\limsup \limsup _{t \rightarrow 0}\left\langle p-f(p), J\left(p-u_{n}\right)\right\rangle \\
& \leq \limsup _{t \rightarrow 0} \limsup _{n \rightarrow \infty}\left\langle u_{t}-f\left(u_{t}\right), J\left(u_{t}-u_{n}\right)\right\rangle \leq 0 .
\end{aligned}
$$

Finally we show that $\lim _{n \rightarrow \infty} u_{n}=p$.

From Lemma 2.2, we have

$$
\begin{aligned}
\left\|u_{n+1}-p\right\|^{2} & =\left\|\lambda_{n}\left(f\left(u_{n}\right)-p\right)+\beta\left(u_{n}-p\right)+\left(1-\beta-\lambda_{n}\right)\left(W_{n} u_{n}-p\right)\right\|^{2} \\
& \leq\left(\beta\left\|u_{n}-p\right\|+\left(1-\beta-\lambda_{n}\right)\left\|u_{n}-p\right\|\right)^{2}+2 \lambda_{n}\left\langle f\left(u_{n}\right)-p, J\left(u_{n+1}-p\right)\right\rangle \\
& =\left(1-\lambda_{n}\right)^{2}\left\|u_{n}-p\right\|^{2}+2 \lambda_{n}\left\langle f\left(u_{n}\right)-f(p), J\left(u_{n+1}-p\right)\right\rangle+2 \lambda_{n}\left\langle f(p)-p, J\left(u_{n+1}-p\right)\right\rangle \\
& \left.\leq\left(1-\lambda_{n}\right)^{2}\left\|u_{n}-p\right\|^{2}+\lambda_{n} \alpha\left\|u_{n}-p\right\|^{2}+\left\|u_{n+1}-p\right\|^{2}\right]+2 \lambda_{n}\left\langle f(p)-p, J\left(u_{n+1}-p\right)\right\rangle
\end{aligned}
$$

Which implies that

$$
\begin{aligned}
\left\|u_{n+1}-p\right\|^{2} & \leq \frac{\left(1-\lambda_{n}\right)^{2}+\lambda_{n} \alpha}{1-\lambda_{n} \alpha}\left\|u_{n}-p\right\|^{2}+\frac{2 \lambda_{n}}{1-\lambda_{n} \alpha}\left\langle f(p)-p, J\left(u_{n+1}-p\right)\right\rangle \\
& \leq\left[1-\frac{2 \lambda_{n}(1-\alpha)}{1-\lambda_{n} \alpha}\left\|u_{n}-p\right\|^{2}\right. \\
& +\frac{2 \lambda_{n}(1-\alpha)}{1-\lambda_{n} \alpha}\left[\frac{1}{1-\alpha}\left\langle f(p)-p, J\left(u_{n+1}-p\right)\right\rangle+\frac{\lambda_{n}}{2(1-\alpha)}\left\|u_{n}-p\right\|^{2}\right] \\
& =\left(1-\gamma_{n}\right)\left\|u_{n}-p\right\|^{2}+\delta_{n} \gamma_{n}
\end{aligned}
$$

Where $\gamma_{n}=\frac{2 \lambda_{n}(1-\alpha)}{1-\lambda_{n} \alpha}, \delta_{n}=\frac{1}{1-\alpha}\left\langle f(p)-p, J\left(u_{n+1}-p\right)\right\rangle+\frac{\lambda_{n}}{2(1-\alpha)}\left\|u_{n}-p\right\|^{2}$

It is easily seen that $\sum_{n=1}^{\infty} \gamma_{n}=\infty$ and $\limsup _{n \rightarrow \infty} \delta_{n} \leq 0$.

Finally we apply Lemma 2.3 to (3.12) to conclude that $\lim _{n \rightarrow \infty} u_{n}=p$. This complete the proof.

\section{References}

1. H. K. Xu, An iterative approach to quadratic optimization, J. Optim. Theory Appl. 241 (2000)

2. H. K. Xu, Viscosity approximation methods for nonexpansive mappings, J. Math. Anal. Appl. 298 (2004) 279-291.

3. K. Goebel, S. Reich, Uniform Convexity, Nonexpansive mappings and Hyperbolic Geometry, Dekker, 1984.

4. S. Atsushiba, W. Takahashi Strong convergence theorems for a finite family of nonexpansive mappings and applications, Indian J. Math. 41 (1999), 435-453.

5. Sh. Banerjee, B. S. Choudhury, Weak and strong convergence theorems of a new iterative process with errors for common fixed points of a finite families of asymptotically nonexpansive mappings in the intermediate sense in Banach spaces. TJMCS, 11(2014), 79-85

6. S. S. Chang, Some problems and results in the study of nonlinear analysis, Nonlinear Anal. 33 (1997) 4197-4208.

7. T. Suzuki, Strong convergence of Krasnoselskii and Mann's type sequences for one-parameter 
Nonexpansive semigroups without Bochner integrals, J. Math. Anal. Appl. 305(2005) 227-239

8. Y. Yao, A general iterative method for a finite family of nonexpansive mappings, Nonlinear Anal. 66 (2007), 2676-2687 